\title{
Sphenopalatine ganglion-targeted low-temperature plasma radiofrequency ablation in the treatment of refractory cluster headache
}

\author{
Nan Wang ${ }^{1,2}$, Zhi Dou ${ }^{1}$, Liangliang $\mathrm{He}^{1}$, Liping Song ${ }^{1}$, Zhaoxuan Guo ${ }^{1}$, Jiaxiang $\mathrm{Ni}^{1}$ \\ ${ }^{1}$ Department of Pain Management, Xuanwu Hospital, Capital Medical University, Beijing, China \\ ${ }^{2}$ Department of Anesthesiology, Civil Aviation General Hospital, Beijing, China
}

Videosurgery Miniinv 2020; 15 (2): 313-318

DOI: https://doi.org/10.5114/wiitm.2020.93529

\begin{abstract}
Introduction: Cluster headache $(\mathrm{CH})$ is a refractory headache. Low-temperature plasma radiofrequency ablation is a relatively novel technique with promising applications in neuropathic pain. It may improve the treatment of $\mathrm{CH}$. Aim: To evaluate the efficacy and safety of sphenopalatine ganglion-targeted low-temperature plasma radiofrequency ablation in the treatment of patients with refractory $\mathrm{CH}$.

Material and methods: A retrospective cohort study including seventy-five patients with refractory cluster headache who underwent computed tomography (CT)-guided sphenopalatine ganglion-targeted low-temperature plasma radiofrequency ablation between January 2015 and December 2017 at the Beijing Xuanwu Hospital was conducted. Results: Effective pain relief rate 3 months after the procedure was $96 \%$ with 40 (53.3\%) patients achieving complete relief; 32 (42.7\%) patients obtained partial relief and 3 (4\%) patients showed no relief. The effective pain relief rate two years after the procedure remained as high as $85.3 \%$ with 29 (38.6\%) achieving complete relief, 35 (46.7\%) partial relief and 11 (14.7\%) no relief. The procedure proved equally effective for both episodic and chronic cluster headache. Complications including facial numbness, masseter weakness, facial hypoesthesia and cheek hematoma were observed, but all were mild and disappeared within 6 months.

Conclusions: CT-guided low-temperature plasma radiofrequency ablation is an effective and safe strategy for refractory cluster headache. For patients who have not responded to conservative treatment, this minimally invasive intervention is a reliable alternative.
\end{abstract}

Key words: cluster headache, sphenopalatine ganglion, low-temperature plasma radiofrequency ablation.

\section{Introduction}

Cluster headache $(\mathrm{CH})$ is a primary headache and is characterized by recurrent attacks of very severe unilateral pain located in and around the orbit that last $15 \mathrm{~min}$ to $3 \mathrm{~h}$. It occurs at a frequency ranging from once every other day to 8 times a day. The severe pain is usually accompanied with ipsilateral cranial autonomic symptoms related to the eyes and nose that present as conjunctival flushing, lacrima- tion, eyelid edema, nasal and/or sinus congestion with rhinorrhea and redness of the face [1]. CH is considered to be one of the worst pains that humans can experience. The intensity of pain is so high that it can induce suicidal thoughts, and so $\mathrm{CH}$ has been named "suicidal headache" [1]. In accordance with the International Classification of Headache Disorder (ICHD-3), $\mathrm{CH}$ is classified into episodic $\mathrm{CH}$ and chronic $\mathrm{CH}$ [2]. Episodic $\mathrm{CH}$ is characterized by regular headache cluster periods that vary in dura-

\section{Address for correspondence}

Prof. Jiaxiang Ni, Department of Pain Management, Xuanwu Hospital, Capital Medical University, 45 Changchun Road, Xicheng District, 100053 Beijing, China, phone: +86 13910743476, fax: +86 010-63175890, e-mail: nijiaxiang@263.net 
tion from 2 weeks to 3 months followed by a relief period that last longer than 2 weeks, while chronic $\mathrm{CH}$ lacks a subsequent relief phase lasting longer than 14 days for a minimum of 1 year [2].

So far, the pathophysiology of $\mathrm{CH}$ remains unclear; however, the sphenopalatine ganglion (SPG) has been considered to play an important role in $\mathrm{CH}$ $[3,4]$. For patients refractory to traditional pharmacologic treatment, SPG targeted invasive treatments, such as SPG block and SPG transection, were considered, but the treatment effects were unsatisfactory and the recurrence rates were high $[5,6]$.

In recent years, SPG targeted radiofrequency thermocoagulation has been reported to be effective in the treatment of $\mathrm{CH}$, but it can cause variable degrees of cheek numbness and hypoesthesia of the palate in patients because the sensory roots in the SPG deriving from the sphenopalatine branch of the maxillary nerve could be irreversibly damaged at $55-70^{\circ} \mathrm{C}[5]$.

SPG targeted pulsed radiofrequency ablation is another procedure reported for the treatment of $\mathrm{CH}$ with less tissue destruction and less painful [7-9]. The disadvantages of pulsed radiofrequency ablation are that there is typically a shorter duration of relief and the procedure may need more frequent repeat treatments, which can lead to a higher cost $[9,10]$.

Low-temperature plasma radiofrequency ablation is a relatively novel technique with promising applications in neuropathic pain. It has been suggested to be effective for the treatment of phantom limb pain [11], trigeminal neuralgia [12] and thoracic neuropathic pain [13], among others. It uses radiofrequency energy through a saline medium to create

Table I. Patient characteristics prior to the surgery

\begin{tabular}{|lc|}
\hline Variable & CH patients \\
\hline Gender, $n(\%):$ & $32(42.7)$ \\
\hline Male & $43(57.3)$ \\
\hline Age at surgery, mean \pm SD [years] & $47.7 \pm 13.7$ \\
\hline Disease duration, mean \pm SD [years] & $12.8 \pm 10.8$ \\
\hline \begin{tabular}{l} 
Side, $n(\%):$ \\
\hline Right
\end{tabular} & $39(52.0)$ \\
\hline Left & $36(48.0)$ \\
\hline
\end{tabular}

plasma iron particles that break molecular bonds in the tissue, which causes the tissue to dissolve at relatively low temperatures. In the present study, SPG-targeted low-temperature plasma radiofrequency ablation was applied in the treatment of refractory $\mathrm{CH}$. Its efficiency and safety were evaluated.

\section{Aim}

To evaluate the efficacy and safety of sphenopalatine ganglion-targeted low-temperature plasma radiofrequency ablation in the treatment of patients with refractory $\mathrm{CH}$.

\section{Material and methods}

\section{General information of patients}

A retrospective study was conducted on 75 patients with $\mathrm{CH}$ who underwent computed tomography (CT)-guided SPG-targeted low-temperature plasma radiofrequency ablation in the Department of Pain Management, Xuanwu Hospital between January 2015 and December 2017. This study was approved by the Ethics Committee of Xuanwu Hospital. Among 75 patients 32 were male and 43 were female. The age ranged from 26 to 84 years (mean age: 48 years). The course of disease ranged from 6 months to 40 years, with mean duration of 13.50 years. 36 cases were on the left side and 39 cases were on the right side (Table I). All cases were in compliance with $\mathrm{CH}$ diagnostic criteria published by the International Pain Association (International Headache Society - IHS) [2], with 54 episodic $\mathrm{CH}$ and 21 chronic $\mathrm{CH}$. All patients were refractory to conventional pharmaceutical treatments.

\section{Procedures}

SPG-targeted low-temperature plasma radiofrequency ablation was performed under $\mathrm{CT}$ guidance. The patient was asked to lie supine on the CT scanner bed. A CT scan was performed to locate the pterygopalatine fossa and the entry route was marked (Photo 1 A). Under local anesthesia with $1 \%$ lidocaine, a $9.5 \mathrm{~cm} 18 \mathrm{G}$ puncture needle was inserted and adjusted to reach the pterygopalatine fossa under CT guidance (Photo $1 \mathrm{~B}$ ). After the tip of the needle was optimally positioned, the needle core was replaced with the plasma ablation needle (DXR-G1100-A185; Xi'an Surgical Medical Technology Co., Ltd., Xi'an, China). To further confirm that 

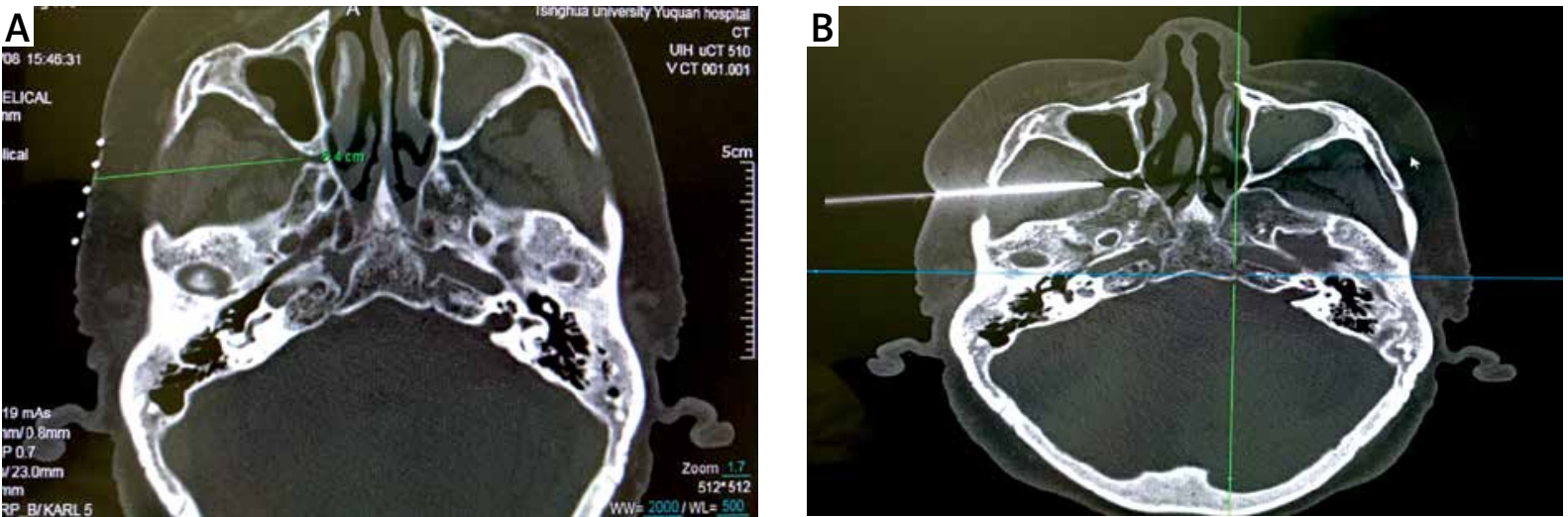

Photo 1. Computed tomography (CT) for confirmation of the needle position. A - CT scan showing location of pterygopalatine fossa. B - CT scan showing the needle gradually reaching the pterygopalatine fossa

the position was adequate, sensory stimulation was performed. The Stimuplex HNS11 Nerve Stimulator (B. Braun Co., Ltd., Melsungen, Germany) was connected to the plasma ablation needle. The nerve stimulator was turned on, adjusted to $2 \mathrm{~Hz}$ and $0.1 \mathrm{~ms}$, and the stimulus intensity was gradually increased to $0.5 \mathrm{mV}$, adjusting the location of the plasma ablation needle until paresthesia was induced along the innervation of the nasal root. If the stimulus was felt in the hard palate (palatal nerve) or the upper tooth (maxillary nerve), the position of the puncture needle needed to be readjusted. The plasma ablation needle was disconnected from the nerve stimulator and connected to the low-temperature plasma generator (SM-D380C; Xian Surgical Medical Technology Co., Ltd., Xian, China). Under intravenous general anesthesia, ablation was performed at $45 \mathrm{~W}$ for $30 \mathrm{~s}$, and repeated after a $30 \mathrm{~s}$ pause.

\section{Data collection and follow-up}

Physical examination was conducted routinely before the procedure and on day 1 and day 3 after the procedure. Follow-up was conducted routinely at 3 and 6 months, and annually after the procedure. The evaluation of pain was performed using the numeric rating scale (NRS) with 0 as no pain and 10 as intolerable pain. The numeric rating scores were recorded on the day before the procedure and 1 and 3 days, 3 and 6 months and annually after the procedure. The efficacy was evaluated based on NRS scores. Complete relief (CR) was defined as no pain (0 points on the NRS), no parasympathetic symptoms without pharmacologic treatment. Partial relief (PR) was defined as a decreased intensity of the attacks resulting in a reduced medication need. No relief was defined as the pain level remaining the same as before the procedure. Effective relief was defined as complete relief or partial relief.

For cheek numbness, the patients were asked whether they had a feeling of numbness. Hypoesthesia during hospitalization was tested through physical examination. A cotton swab was used to lightly touch patients' cheeks on both sides and the patients were asked whether the cheeks on both sides had the same feeling. For muscle weakness or atrophy, the patients were checked and asked whether their faces were symmetrical and whether there was muscle weakness when chewing and biting.

\section{Statistical analysis}

SPSS18.0 statistical software was used for data analysis in this study. Data are presented as the mean $\pm \mathrm{SD} . P<0.05$ indicated that there was statistical significance.

\section{Results}

\section{Efficacy in pain relief and pain recurrence}

Obvious pain relief was observed after the procedure. NRS scores dramatically decreased from $7.97 \pm 1.25$ before the procedure to $1.14 \pm 1.42,1.30$ $\pm 2.05,1.50 \pm 2.25,1.71 \pm 2.30,1.80 \pm 2.42$ and 1.83 \pm 2.53 at 1 and 3 days, 3 and 6 months, 1 and 2 years after the procedure, respectively (Figure 1). There was a significant difference between NRS score before the procedure and NRS score at any time point after the procedure $(p<0.001$ in all). Since the episodic $\mathrm{CH}$ patients had different time of pain free 


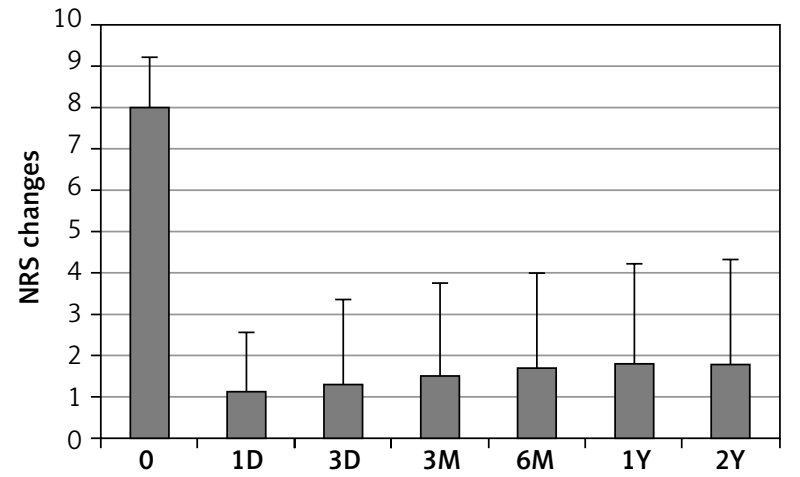

Figure 1. NRS score differences before and after the procedure

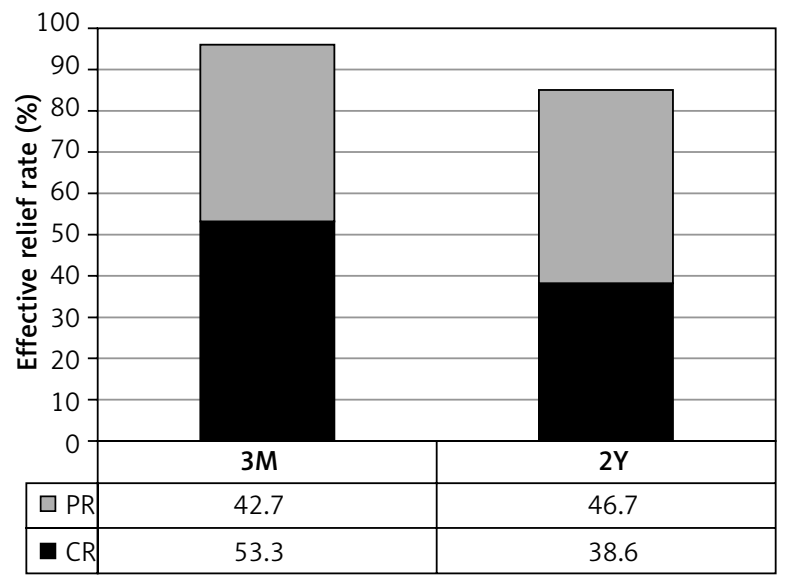

Figure 2. Effective relief rates at 3 months and 2 years after the procedure

$C R$-complete relief, $P R$ - partial relief.

interval between pain cluster periods, the effective relief at 3 months after the procedure was considered as the initial treatment outcome. The effective relief rate at 3 months after the procedure was $96 \%$ with 40 (53.3\%) patients achieving complete relief with no any pain and 32 (42.7\%) patients obtaining partial relief with less severe pain (NRS scores, mostly 2-4) (Figure 2). Three (4\%) patients showed no relief. These three no pain relief cases were considered as invalid ablation. All patients received at least 2 years of follow-up. The effective relief rate at 2 years after the procedure remained as high as $85.3 \%$ with 29 (38.6\%) obtaining complete relief and 35 (46.7\%) partial relief (Figure 2). Eleven (14.7\%) patients showed no relief including 3 previous invalid ablation cases and $8(10.6 \%)$ recurrent cases. The recurrence occurred at different time points after the procedure including 6 months (3 patients), 1 year
(3 patients) and 2 years (2 patients). Among $75 \mathrm{CH}$ patients, 54 had episodic pain and 21 had chronic clustered pain. The effective relief rate at 2 years after the procedure in chronic clustered cases was $85.7 \%$ with 8 (38.1\%) cases of complete relief, $10(47.6 \%)$ of partial relief and $3(14.2 \%)$ of no relief. This outcome was not significantly different compared to the total group. Therefore SPG low-temperature plasma radiofrequency ablation was equally effective for both episodic and chronic clustered $\mathrm{CH}$.

\section{Complications}

Twenty-nine (38.7\%) patients had mild and moderate degrees of facial numbness immediately after ablation. Facial numbness gradually improved and disappeared within 6 months with duration of 10.5 \pm 7.48 weeks. Additional post-procedure complications including mild masseter weakness ( 2 cases, $2.7 \%$ ), cheek hypoesthesia (9 cases, $12 \%$ ) and cheek hematoma (2 cases, $2.7 \%$ ) were observed and all disappeared within 3 months.

\section{Discussion}

The sphenopalatine ganglion has been proven to play an important role in $\mathrm{CH}$. For patients who have not responded to drug treatment or cannot tolerate the treatment-related side effects, SPG-targeted invasive treatments are taken into consideration. In recent years, it has been reported that SPG radiofrequency ablations, both thermal and pulsed, are relatively quick, cost-effective and safe procedures for treating $\mathrm{CH}$. Radiofrequency thermocoagulation (RFTA), also known as radiofrequency neurotomy, is a method used to destroy painful nerves with $70^{\circ} \mathrm{C}$ to $90^{\circ} \mathrm{C}$ heat. The RFTA device uses high frequency (ranges: $300-500 \mathrm{kHz}$ ) to create charged molecular oscillation which generates heat by the friction of ions and radio waves. In conventional RFTA, the special RFTA needle generates a 5-15 mm electric field that ultimately increases the temperature of the affected tissue. This temperature produces local tissue damage and loss of myelinated fibers. When the needle tip heats to $80^{\circ} \mathrm{C}$ for 60 to $90 \mathrm{~s}$, it reliably produces an 8-10 mm affected area. RFTA is effective for pain relief, but it can cause variable degrees of cheek numbness or dysesthesia of the palate, maxilla, or post pharynx in patients because the sensory roots in the SPG coming from the sphenopalatine branch of the maxillary nerve could be irreversibly damaged by heat. Pulsed radiofrequency 
ablation (PRFA) is another radiofrequency technique. It is performed with similar short 20-ms pulses every $0.5 \mathrm{~s}$, which allows time for the tissue to cool and does not exceed the target temperature of $42^{\circ} \mathrm{C}$. The advantage of PRFA is that there is less tissue destruction and that it is less painful. The disadvantages of PRFA are that there is typically a shorter duration of relief and the patients may need more frequent repeat treatments, which can lead to a high cost [7-9].

Low-temperature plasma radiofrequency ablation is a relatively novel minimally invasive technique with promising applications in neuropathic pain [11-13]. Its working principle is to make the local tissue around the electrode tip form a plasma field by $100 \mathrm{kHz}$ radio frequency energy; a thin layer plasma of 50-100 $\mu \mathrm{m}$ with a large number of ion particles carrying enough kinetic energy is formed, ablating target tissues by cutting off organic molecular bonds in tissues at low temperatures of about $40-50^{\circ} \mathrm{C}$. Therefore, the principle action of plasma radiofrequency ablation is based on molecular dissociation at low temperature rather than thermal damage as in radiofrequency thermocoagulation $[14,15]$.

Salgado-Lopez et al. [7] published their results on the safety and efficacy of SPG radiofrequency ablation (both RFTA and PRFA) for treating chronic $\mathrm{CH}$. They followed 37 patients for a mean follow-up of 68.1 months (range: $15-148$ months). Five (13.5\%) of the patients experienced complete clinical relief of both pain and parasympathetic symptoms, 21 (56.8\%) patients had partial and transient relief, and 11 (29.7\%) patients did not improve. Chen et al. [9] reported their results of SPG pulsed radiofrequency (PRFA) in the treatment of $\mathrm{CH}$. In total 59 patients underwent 106 PRFA procedures within an observational period of 24 months (range: 3-108 months). Effective remission at 30 days after the PRFA procedure was observed in $95.6 \%$ and $64.3 \%$ of patients with refractory episodic and chronic $\mathrm{CH}$, respectively. However, high recurrence was observed and repeated PRFA procedures were performed for recurrent patients.

In this study, CT-guided SPG low-temperature plasma radiofrequency ablation was applied in the treatment of 75 patients with refractory $\mathrm{CH}$. Obvious pain relief was observed immediately after the procedure. The effective relief rate at 3 months after the procedure was $96 \%$. Forty (53.3\%) patients achieved complete relief; 32 (42.7\%) patients obtained partial relief and 3 (4\%) patients showed no relief. These three no pain relief cases were considered as inval- id ablation. All patients completed at least 2 years of follow-up. The effective relief rate at 2 years after the procedure remained as high as $85.3 \%$ with $29(38.6 \%)$ patients achieving complete relief and 35 (46.7\%) patients obtaining partial relief. Eleven (14.7\%) patients showed no relief including 3 previous invalid cases and 8 (10.6\%) recurrent cases. These results indicated that SPG-targeted low-temperature plasma radiofrequency ablation is effective for the treatment of refractory $\mathrm{CH}$.

It has been reported that chronic $\mathrm{CH}$ showed more resistance to the treatment of pulsed radiofrequency ablation compared to episodic $\mathrm{CH}$ [7-9]. In this study, among $75 \mathrm{CH}$ patients, 21 cases were chronic $\mathrm{CH}$. The effective relief rate at 2 years after the procedure for 21 chronic $\mathrm{CH}$ was $85.7 \%$ with $8(38.1 \%)$ complete relief, 10 (47.6\%) partial relief and $3(14.2 \%)$ no relief. This result is similar to the outcome of total group. Our data demonstrated that SPG-targeted low-temperature plasma radiofrequency ablation was equally effective for both episodic and chronic clustered $\mathrm{CH}$.

The low-temperature plasma radiofrequency ablation procedure causes fewer complications compared to radiofrequency thermocoagulation because it uses plasma ion particles to cut off organic molecular bonds in tissues at low temperatures of about $40-50^{\circ} \mathrm{C}$ rather than $70^{\circ} \mathrm{C}$ to $90^{\circ} \mathrm{C}$ as in radiofrequency thermocoagulation. In the comparative study of Gasserian ganglion-targeted low-temperature plasma radiofrequency ablation vs. radiofrequency thermocoagulation in the treatment of trigeminal neuralgia performed in our department, facial numbness in the plasma group (44\%) was dramatically lower than that in the thermocoagulation group (70.9\%) [12]. In this study, 29 (38.7\%) patients had mild to moderate degrees of facial numbness immediately after ablation. Facial numbness gradually improved and disappeared within 6 months with duration of $10.5 \pm 7.48$ weeks. Additional post-procedure complications included masseter weakness (2 cases, $2.7 \%$ ), cheek hypoesthesia (9 cases, $12 \%$ ) and cheek hematoma (2 cases, 2.7\%). All these complications were mild and disappeared within 3 months. No severe complications were observed in this study.

\section{Conclusions}

CT-guided SPG-targeted low-temperature plasma radiofrequency ablation is an effective and safe 
procedure for refractory $\mathrm{CH}$. For patients who have not responded to conservative treatment, this minimally invasive intervention is a reliable alternative.

\section{Conflict of interest}

The authors declare no conflict of interest

\section{References}

1. Rozen TD, Fishman RS. Cluster headache in the United States of America: demographics, clinical characteristics, triggers, suicidality, and personal burden. Headache 2012; 52: 99-113.

2. Headache Classification Committee of the International Headache Society (IHS). The international classification of headache disorders. Cephalalgia 2018; 38: 1-211.

3. Robbins MS, Robertson CE, Kaplan E, et al. The sphenopalatine ganglion: anatomy, pathophysiology, and therapeutic targeting in headache. Headache 2016; 56: 240-58.

4. Narouze SN. Role of sphenopalatine ganglion neuroablation in the management of cluster headache. Curr Pain Headache Rep 2010; 14: 160-3.

5. Tolba R, Weiss AL, Denis DJ. Sphenopalatine ganglion block and radiofrequency ablation: technical notes and efficacy. Ochsner J 2019; 19: 32-7.

6. Fontaine D, Santucci S, Lanteri-Minet M. Managing cluster headache with sphenopalatine ganglion stimulation: a review. J Pain Res 2018; 11: 375-81.

7. Salgado-Lopez L, de Quintana-Schmidt C, Belvis Nieto R, et al. Efficacy of sphenopalatine ganglion radiofrequency in refractory chronic cluster headache. World Neurosurg 2019; 122: e262-9.

8. Fang L, Jingjing L, Ying S, et al. Computerized tomography-guided sphenopalatine ganglion pulsed radiofrequency treatment in 16 patients with refractory cluster headaches: twelve- to 30-month follow-up evaluations. Cephalalgia 2016; 36: 106-12.

9. Chen Z, Ren H, Zhao CM, Luo F. Long-term outcomes of computerized tomography-guided sphenopalatine ganglion-targeted pulsed radiofrequency for refractory cluster headache. Pain Pract 2019; 11 doi:10.1111/papr.12853.

10. Bendersky DC, Hem SM, Yampolsky CG. Unsuccessful pulsed radiofrequency of the sphenopalatine ganglion in patients with chronic cluster headache and subsequent successful thermocoagulation. Pain Pract 2015; 15: E40-5.

11. Li H, Li Y, Guo Z, et al. Low-temperature plasma radiofrequency ablation in phantom limb pain: a case report. Brain Circ 2018; 4: 62-4.

12. Li Y, Guo Y, Yang LQ, Ni JX. Comparison of the short-term outcomes after low-temperature plasma radiofrequency ablation (coblation) in the Gasserian ganglion for the treatment of idiopathic trigeminal neuralgia. J Pain Res 2019; 12: 1235-42.

13. Yang LQ, Gong WY, Wang XP, et al. Computed tomography-guided percutaneously controlled ablation of the thoracic paravertebral nerve due to thoracic neuropathic pain. Pain Pract 2017; 17: 792-9.

14. Wang Z, Zhang Y, Cui $\mathrm{H}$, et al. Endoscopic low-temperature plasma radiofrequency ablation for laryngeal plexiform neuro- bromatosis-1 in an infant: case report and review of the literature. Technol Health Care 2016; 24: 775-84.

15. Sun D, Li Q, Tang Y, et al. Comparison of coblation annuloplasty and radiofrequency thermocoagulation for treatment of lumbar discogenic pain. Medicine 2017; 96: e8538.

Received: 3.02.2020, accepted: 16.02.2020 